\title{
NIVEL DE CONOCIMIENTOS SOBRE INFECCIONES DE TRASMISIÓN SEXUAL - VIH/SIDA Y SUS MEDIDAS PREVENTIVAS EN ESTUDIANTES DE CIENCIAS DE LA SALUD DE ICA 2017
}

Level Of Knowledge On Sexual Transmission Infections (Its) Hiv / Aids And Its Preventive Measures In Students Of Ica Health Sciences 2017

\section{ARTÍCULO ORIGINAL}

Jesús Nicolasa Meza-León ${ }^{1, a, b}$, Jaihro Andres Conislla-Monterola ${ }^{2, b}$, Mery Liset Huarancca-Gavilán ${ }^{2, b}$ Karen Denisse Soto-Meneses ${ }^{2, b}$

1. Médico Ginecóloga

2. Medicina Humana

a. Hospital Regional de Ica

b. Facultad de Medicina Humana, Universidad Nacional San Luis Gonzaga de Ica

\section{Correspondencia:}

Jaihro Andres Conislla Monterola

Domicilio: San Idelfonso las

Malvinas 247, Tinguiña - Ica.

Telefono: 926580552

Correo Electrónico:

Jaihro_15_03@hotmail.com

Contribuciones De Autoría: MLJN, CMJA, HGAJ, SMKD participaron en el diseño del estudio, el análisis de los datos, revisaron críticamente el artículo y aprobaron la versión final.

Conflicto De Intereses: No declarados.

Financiamiento: Autofinanciado.

Recibido: 05-11-2017

Aceptado: 20-11-2017

Publicado: 20-12-2017

\section{Como Citar}

Meza-Leon J, Conislla Monterola J, Huarancca Gavilan L, Soto Meneses K. nivel de conocimientos sobre infecciones de trasmisión sexual - vih/sida y sus medidas preventivas en estudiantes de ciencias de la salud de Ica 2017. Rev méd panacea. 2017;6 (3) 104 110

\begin{abstract}
RESUMEN:
Objetivo: Determinar el nivel de conocimientos sobre las infecciones de trasmisión sexual (ITS) - VIH-SIDA y sus medidas preventivas en estudiantes de Medicina Humana, Enfermería y Obstetricia de la Universidad Nacional San Luis Gonzaga de Ica. Material y Metodos: Se realizó un estudio descriptivo, prospectivo, transversal y comparativo con un muestreo probabilístico, en total se encuestaron 290 estudiantes. La técnica de recolección de datos fue la encuesta y como instrumento el cuestionario. Los datos fueron digitados y analizados en el programa estadístico SPSS $21.0 \mathrm{G}$. Para el análisis inferencial, se planteó ver el grado de asociación de las variables estudiadas sobre la base del cálculo de la prueba Chi-cuadrado, con un nivel de confianza (IC) del 95\%. Un valor $\mathrm{p} \leq 0.05$ se consideró significativo. Resultados: En forma global predomina el conocimiento medio en Obstetricia con $81.8 \%$ y en Enfermería con $76.8 \%$, mientras que en Medicina predomina el nivel alto con 45.6\%. En la dimensión del conocimiento, predomina el nivel bajo en Enfermería con $78.51 \%$ y en Obstetricia con $50.9 \%$, mientras que en Medicina predomina el nivel alto con $36.8 \%$. En la dimensión de las medidas preventivas se observa que predomina el nivel de conocimiento alto en general. Conclusiones: Los estudiantes de Medicina presentan un mejor nivel de conocimientos, seguido de Obstetricia y por último los de Enfermería.
\end{abstract}

Palabras Claves: Conocimiento, VIH, sexo seguro, SIDA, sexualidad. (Fuente: BeCS-BIREME)

\begin{abstract}
:
Objetive: To determine the level of knowledge about sexually transmitted infections (STIs) - HIV-AIDS and its preventive measures in students of Human Medicine, Nursing and Obstetrics, National University San Luis Gonzaga de Ica. Material And Methods: A descriptive, prospective, cross-sectional and comparative study was carried out with a probabilistic sampling, in total 290 students were surveyed. The data collection technique was the survey and as a questionnaire instrument. The data were entered and analyzed in the statistical program SPSS $21.0 \mathrm{G}$. For the inferential analysis, it was proposed to see the degree of association of the variables studied on the basis of the Chi-square test, with a confidence level $(\mathrm{Cl})$ Of $95 \%$. A p value $\leq 0.05$ was considered significant. Results: Overall, the average knowledge in Obstetrics is $81.8 \%$, and in Nursing it is $76.8 \%$, whereas in Medicine, the high level is predominant with $45.6 \%$. In the knowledge dimension, the low level in Nursing predominates with $78.51 \%$ and in Obstetrics with $50.9 \%$, whereas in Medicine the high level predominates with $36.8 \%$. In the dimension of the preventive measures it is observed that the high level of knowledge predominates in general. Conclusions: Medical students present a better level of knowledge, followed by Obstetrics and finally Nursing.
\end{abstract}

Keywords: Knowledge, sexually transmitted infections, sexuality, sexual ethics. 


\section{INTRODUCCIÓN}

Las infecciones de transmisión sexual (ITS) constituyen un grave problema de salud a nivel mundial ya que depende del comportamiento humano, es por eso que la prevención y la promoción de salud en esta esfera rebasan los marcos de la salud para no ser tan solo un problema sino también una responsabilidad de todos los sectores de la sociedad (1). La influencia de diversos canales de socialización de la sexualidad como la religión, la familia y las instituciones educativas determinan un nivel de conocimientos en el ámbito de la sexualidad (2). Sin embargo, cuando la información es obtenida de fuentes nada fiables como revistas, películas o información por parte de los compañeros se presenta una visión muy reduccionista de la sexualidad (3). El incremento de estas infecciones se atribuye a diferentes factores entre los cuales figuran: cambios en la conducta sexual y social, así como la tendencia actual a una mayor promiscuidad sexual, aumento considerablemente el consumo de alcohol u otras sustancias y una falta de interés por adquirir conocimientos sobre estos aspectos (4). Al respecto se han realizado diversos estudios tanto en el ámbito internacional como nacional entre ellos; Camacho J. (4), quien realizó un estudio en el año 2013 titulado: Relación entre el nivel de conocimientos sobre las infecciones de trasmisión sexual (ITS) y la actitud hacia conductas sexuales de riesgo en estudiantes de la facultad de medicina UNMSM, donde encontró que el mayor porcentaje de estudiantes tienen conocimiento medio acerca de las ITS y actitudes de rechazo hacia las conductas sexuales de riesgo. Quispe Y. y Ramos K. (5), en el 2015 en su trabajo denominado: Conocimiento sobre VIH-SIDA y actitud frente al uso de preservativo en estudiantes de la Facultad de Enfermería de la Universidad Nacional de Huancavelica, donde sus resultados indicaron que más de la mitad (69,6\%) de estudiantes presentan conocimiento alto sobre VIH-SIDA y actitud positiva frente al uso de preservativo. Ante todo esto Definimos como Infecciones de Transmisión Sexual - término recomendado por la OMS como todas aquellas infecciones que se transmiten a través del contacto sexual, durante las relaciones sexuales penetrativas del pene o la lengua en la vagina, el ano o la cavidad oral con una persona infectada. Algunas ITS también son transmitidas de madre a hijo y/o a través del contacto sanguíneo (6). La conducta sexual de riesgo se estudia con frecuencia en instituciones educativas de nivel medio y superior porque se le asocia con dos factores que pueden interrumpir el proceso formativo y el proyecto de vida de los estudiantes $(7,8,9)$. Las prácticas de riesgo pueden entenderse como "la exposición del individuo a una situación que pueda ocasionar daños a su salud o la de otra persona, especialmente a través de la posibilidad de contaminación por enfermedades sexualmente transmisibles como el SIDA" (10, 11, 12). Mundialmente hay 340 millones de ITS nuevas por año, en edades de 15 y 49 años, las tasas más altas los jóvenes, cada año ocurren, por lo menos 111 millones de casos nuevos curables en este grupo, si no se tratan, pueden causar esterilidad, enfermedad inflamatoria pélvica, embarazo ectópico y cáncer $(13,14)$. Actualmente, si bien se ha producido un aumento en la utilización de preservativos, también han aumentado las ITS, se ha experimentado un adelanto en la edad de inicio de relaciones sexuales, un aumento de embarazos en adolescentes y de interrupciones voluntarias de embarazos (15, 16). Además se evidencia como problemas relevantes a las prácticas sexuales, las altas tasas de fecundidad, el aborto provocado y el elevado consumo de alcohol (17). En este sentido, se ha observado cómo el uso de alcohol reduce las habilidades para negociar el sexo seguro, influye en que no se utilice preservativo, que es una de las variables que mejor predice las conductas sexuales de riesgo $(18,19)$. La Salud sexual y reproductiva, constituye una preocupación esencial en la formación humana, especialmente en la juventud, el no tener políticas y estrategias que fomenten una sexualidad plena, los hace vulnerables ante la coerción, el abuso y la explotación sexual, el embarazo no planificado, el aborto en condiciones de riesgo y las
ITS, incluyendo el virus de la inmunodeficiencia humana (VIH) y el sida (20). Los profesionales de la salud tienen la misión de realizar actividades para la prevención de las enfermedades en salud sexual y reproductiva, tal es el caso de la prevención del embarazo no deseado, del aborto clandestino, del embarazo adolescente, entre otros. Es esencial que el personal asistencial enfatice la importancia de la sexualidad, mediante la educación sexual, tanto en conocimientos como en prácticas y actitudes. Por ello que el presente estudio tiene como objetivo principal: Determinar el nivel de conocimientos sobre las infecciones de trasmisión sexual (ITS) VIH-SIDA y sus medidas preventivas en estudiantes de Medicina Humana, Enfermería y Obstetricia de la Universidad Nacional San Luis Gonzaga de Ica. Además, el presente estudio servirá como un aporte científico a la comunidad médica y ayudará a la realización de otras investigaciones respecto a este tema, pues muy pocos estudios han sido llevados a cabo. ${ }^{\circ}$

\section{MATERIAL Y MÉTODOS}

Se aplicó el método prospectivo y observacional de nivel descriptivo, transversal y comparativo, considerando la información sobre las Infecciones de Transmisión Sexual y sus Medidas Preventivas. La población está conformada por todos los estudiantes de las facultades de Medicina Humana, Enfermería y Obstetricia, siendo un total de 1230 estudiantes. En relación a su distribución se tiene que en Medicina Humana son 500 estudiantes, enfermería 530 y en obstetricia 200 respectivamente. Por lo tanto se realizó un muestreo aleatorio simple para conformar la muestra de 290 estudiantes, además se estratifico por facultad, correspondiendo esto a la presente proporción: 114 en la facultad de Medicina Humana, 121 de la facultad de Enfermería y 55 de la facultad de Obstetricia, todos ellos correspondientes del $3^{\circ}$ y $4^{\circ}$ año de estudios, con un promedio de edad entre los 16 a 25 años, cuya participación fue de carácter voluntario. Se realizó el contacto con la institución universitaria haciendo de conocimiento a los Decanos y Director del Departamento Académico de cada facultad, a su vez, a los participantes con el fin de explicar los propósitos, objetivos y procedimientos relacionados con la investigación; también se les informo sobre el contenido de los instrumentos, la forma y el tiempo requerido para su aplicación, así como el manejo confidencial que se le daría a la información suministrada. Luego se diligencio los consentimientos informados y se inició el proceso de aplicación del instrumento. El tiempo de aplicación del cuestionario fue de 30 minutos. Se aplicó el método de la encuesta y la técnica del cuestionario dividido en dos partes:

- $\quad$ Cuestionario sobre el nivel de conocimientos sobre las infecciones de transmisión sexual VIH-SIDA.

- $\quad$ Cuestionario sobre el nivel de conocimientos sobre las medidas preventivas de las infecciones de transmisión sexual $\mathrm{VIH} / \mathrm{SIDA}$.

El instrumento que se utilizó para la recolección de datos es un cuestionario que permitió obtener información de la fuente directa a través de ítems cerrados y abiertos (dicotómicos y policotómicos). Dicho instrumento consta de introducción, instrucciones, datos generales de los participantes y la sección de información específica que explora los conocimientos sobre las Infecciones de Transmisión Sexual-VIH/SIDA y sus medidas preventivas. El cuestionario consta de 27 ítems, 23 de los cuales están referidos a explorar los conocimientos sobre las ITS, cada respuesta correcta obtuvo 1 punto y cada respuesta incorrecta o puntos. Se realizó una revalidación del instrumento que se determinó mediante Juicio de Expertos, participaron 3 profesionales Gineco-obstetras (Área de consultorio externo y emergencia) del Hospital Regional de Ica. Se determinó la confiabilidad del cuestionario con la prueba estadística de K-RICHARDSON siendo de 0,95. Para el análisis de los datos obtenidos se utilizó la Escala de Stanones para determinar el nivel de conocimientos, obteniendo nivel de conocimiento alto, medio y bajo. Dichos resultados se presentará a través de cuadros y gráficos 
haciendo uso del paquete estadístico SPSS 21.0 G, contrastados con el marco teórico y con trabajos de investigación similares. Para el análisis inferencial, se planteó ver el grado de asociación de las variables estudiadas sobre la base del cálculo de la prueba Chi-cuadrado, con un nivel de confianza (IC) del 95\%. Un valor $\mathrm{p} \leq$ 0.05 se consideró significativo.

\section{RESULTADOS}

De los estudiantes encuestados se observa que en el 88.3\% (256) sus edades fluctúan entre 20 a 25 años, seguido del $11.7 \%$ (34) cuyas edades están en el rango de 16 a 19 años. En relación al Sexo, se observa que el $79 \%$ (299) estudiantes son de sexo femenino y el $21 \%$ (61) masculino, es clara la predominancia del sexo femenino. En cuanto al Estado Civil se obtuvo que el 100\% (176) estudiantes son solteros.

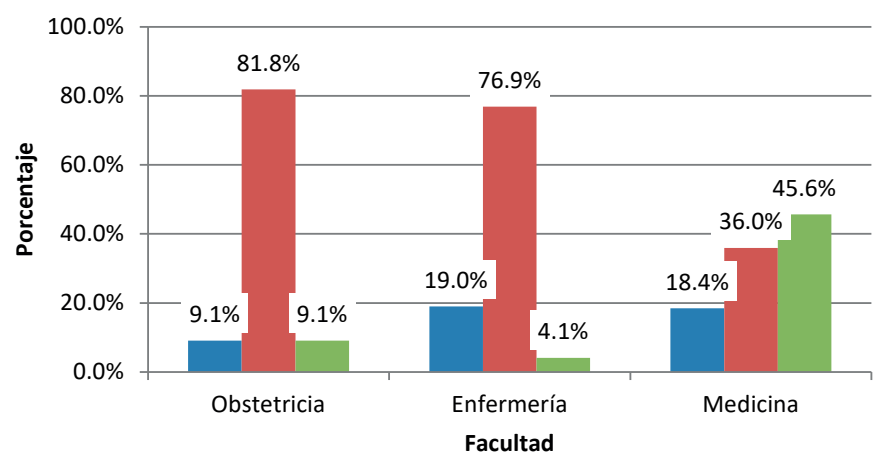

- Conocimiento Bajo Conocimiento Medio Conocimiento Alto

Fuente: Encuesta

Figura 1: Nivel de conocimientos sobre las infecciones de transmisión sexual - vih/sida y sus medidas preventivas en los estudiantes de la unslg, según facultad - 2017

En la figura 1, se observa que en la facultad de Medicina predomina el nivel de conocimiento alto con un $45.5 \%$ (52), seguido de un nivel de conocimiento medio con $35.9 \%$ (41), luego con $18.4 \%$ (21) de nivel de conocimiento bajo. En Obstetricia predomina el nivel de conocimiento medio con $81.8 \%$ (45), seguida de un nivel de conocimiento alto y bajo de $9 \%$ (5). En la facultad de Enfermería el nivel de conocimiento medio representa un $76.8 \%$ (93), luego un nivel de conocimiento bajo de $19 \%$ (23), seguida de un $4.2 \%$ (5) con un nivel de conocimiento alto.

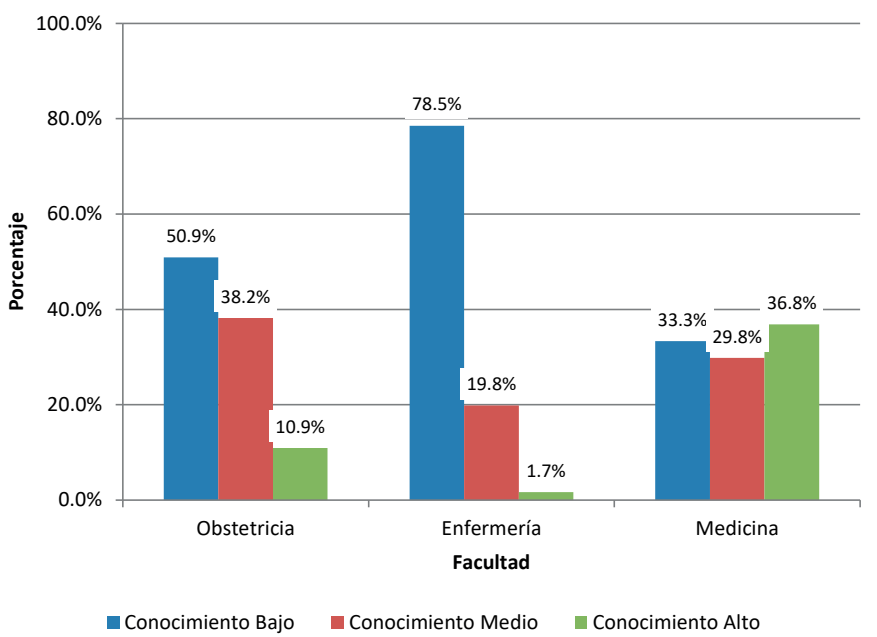

Fuente: Encuesta

Figura 2: nivel de conocimientos sobre las infecciones de transmisión sexual - vih/sida en los estudiantes de la unslg, según facultad, 2017.
En cuanto al nivel de conocimientos según dimensiones en la figura 2 se observa que en la facultad de Medicina predomina el nivel de conocimiento alto con un $36.8 \%$ (42), seguido de un nivel de conocimiento bajo con $33.34 \%$ y luego con un $29.82 \%$ de nivel de conocimiento medio. Se tiene que en la facultad de Enfermería predomina el nivel de conocimiento bajo con un $78.5 \%$ (95), seguido por un nivel de conocimiento medio con un $19.8 \%$ (24) y con $1.7 \%$ (2) de nivel de conocimiento alto. Con respecto a la facultad de Obstetricia presenta un 50.9\% (28) de nivel de conocimiento bajo, seguido de un nivel de conocimiento medio con un $38.2 \%$ (21), luego con un $10.9 \%$ (6) de nivel de conocimiento alto.

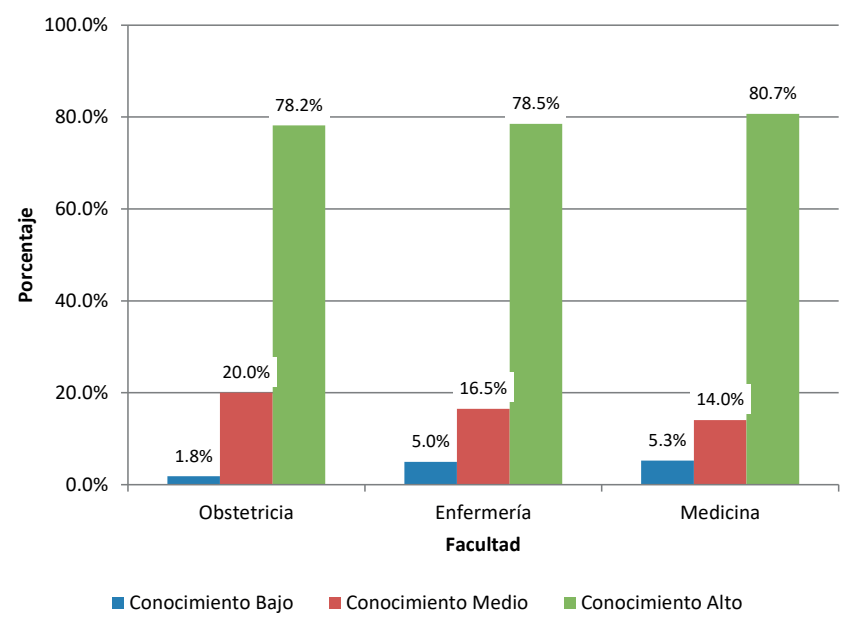

Fuente: Encuesta

Figura 3: nivel de conocimientos sobre las medidas preventivas de las infecciones de transmisión sexual - vih/sida en los estudiantes de la unslg según facultad, 2017.

En cuanto a la Figura 3 se observa que predomina el nivel de conocimiento alto en todas las facultades, siendo en Medicina de 80.7\% (92), en Enfermería de 78.5\% (95) y en Obstetricia de $78.2 \%$. 
Tabla 1: Áreas críticas en el conocimiento sobre its - vih/sida en los estudiantes de la unslg, según facultad, 2017

\begin{tabular}{|c|c|c|c|c|c|c|c|c|c|c|c|c|c|c|c|c|}
\hline & \multicolumn{16}{|c|}{ Facultad } \\
\hline & \multicolumn{4}{|c|}{ Obstetricia } & \multicolumn{4}{|c|}{ Enfermería } & \multicolumn{4}{|c|}{ Medicina } & \multicolumn{4}{|c|}{ Total } \\
\hline & \multicolumn{2}{|c|}{ Incorrecto } & \multicolumn{2}{|c|}{ Correcto } & \multicolumn{2}{|c|}{ Incorrecto } & \multicolumn{2}{|c|}{ Correcto } & \multicolumn{2}{|c|}{ Incorrecto } & \multicolumn{2}{|c|}{ Correcto } & \multicolumn{2}{|c|}{ Incorrecto } & \multicolumn{2}{|c|}{ Correcto } \\
\hline & $\mathrm{N}$ & $\%$ & $\mathrm{~N}$ & $\%$ & $N$ & $\%$ & $N$ & $\%$ & $N$ & $\%$ & $\mathrm{~N}$ & $\%$ & $N$ & $\%$ & $N$ & $\%$ \\
\hline reg.1 & 7 & $12,7 \%$ & 48 & $87,3 \%$ & 11 & $9,1 \%$ & 110 & $90,9 \%$ & 31 & $27,2 \%$ & 83 & $72,8 \%$ & 49 & $16,9 \%$ & 241 & 83,1 \\
\hline$\overline{\text { Preg. } 2}$ & 0 & $0,0 \%$ & 55 & $100,0 \%$ & 6 & $5,0 \%$ & 115 & $95,0 \%$ & 5 & $4,4 \%$ & 109 & $\%$ & 11 & $3,8 \%$ & 279 & 6 \\
\hline Preg.3 & 6 & $10,9 \%$ & 49 & $89,1 \%$ & 16 & $13,2 \%$ & 105 & $86,8 \%$ & 23 & $20,2 \%$ & 91 & $79,8 \%$ & 45 & $15,5 \%$ & 245 & $84,5 \%$ \\
\hline Preg.4 & 0 & $0,0 \%$ & 55 & $100,0 \%$ & 7 & $5,8 \%$ & 114 & $94,2 \%$ & 3 & $2,6 \%$ & 111 & $97,4 \%$ & 10 & $3,4 \%$ & 280 & 96,6 \\
\hline Preg.5 & 4 & $7,3 \%$ & 51 & $92,7 \%$ & 49 & $40,5 \%$ & 72 & $59,5 \%$ & 5 & $4,4 \%$ & 109 & $95,6 \%$ & 58 & $20,0 \%$ & 232 & $80,0 \%$ \\
\hline Preg.6 & 55 & $100,0 \%$ & 0 & $0,0 \%$ & 121 & $100,0 \%$ & 0 & $0,0 \%$ & 65 & $\%$ & 49 & $43,0 \%$ & 241 & . & 49 & $16,9 \%$ \\
\hline$\overline{\text { Preg.7 }}$ & 55 & $100,0 \%$ & 0 & $0,0 \%$ & 121 & $100,0 \%$ & 0 & $0,0 \%$ & 91 & $79,8 \%$ & 23 & $20,2 \%$ & 267 & a & 23 & $7,9 \%$ \\
\hline Preg.8 & 47 & $85,5 \%$ & 8 & $14,5 \%$ & 109 & $90,1 \%$ & 12 & $9,9 \%$ & 59 & $51,8 \%$ & 55 & $48,2 \%$ & 215 & 6 & 75 & $25,9 \%$ \\
\hline Preg.9 & 55 & $100,0 \%$ & 0 & $0,0 \%$ & 121 & $100,0 \%$ & 0 & $0,0 \%$ & 114 & $100,0^{\circ}$ & 0 & $0,0 \%$ & 290 & $100,0 \%$ & 0 & $0,0 \%$ \\
\hline Preg.10 & 44 & $80,0 \%$ & 11 & $20,0 \%$ & 104 & $86,0 \%$ & 17 & $14,0 \%$ & 78 & $68,4 \%$ & 36 & $31,6 \%$ & 226 & $77,9 \%$ & 64 & $22,1 \%$ \\
\hline Preg.11 & 52 & $94,5 \%$ & 3 & $5,5 \%$ & 112 & $92,6 \%$ & 9 & $7,4 \%$ & 93 & 0 & 21 & $18,4 \%$ & 257 & $88,6 \%$ & 33 & $11,4 \%$ \\
\hline Preg.12 & 0 & $0,0 \%$ & 55 & $100,0 \%$ & 56 & $46,3 \%$ & 65 & $53,7 \%$ & 3 & $2,6 \%$ & 111 & $97,4 \%$ & 59 & $20,3 \%$ & 231 & $79,7 \%$ \\
\hline Preg.13 & 46 & $83,6 \%$ & 9 & $16,4 \%$ & 66 & $54,5 \%$ & 55 & $45,5 \%$ & 64 & $56,1 \%$ & 50 & $43,9 \%$ & 176 & $60,7 \%$ & \begin{tabular}{|l|}
114 \\
\end{tabular} & $39,3 \%$ \\
\hline Preg.14 & 6 & $10,9 \%$ & 49 & $89,1 \%$ & 56 & $46,3 \%$ & 65 & $53,7 \%$ & 15 & $13,2 \%$ & 99 & $86,8 \%$ & 77 & $26,6 \%$ & 213 & $73,4 \%$ \\
\hline Preg.15 & 53 & $96,4 \%$ & 2 & $3,6 \%$ & 118 & $97,5 \%$ & 3 & $2,5 \%$ & 101 & $88,6 \%$ & 13 & $11,4 \%$ & 272 & 93 & 18 & $6,2 \%$ \\
\hline Preg.16 & 38 & $69,1 \%$ & 17 & $30,9 \%$ & 85 & $70,2 \%$ & 36 & $29,8 \%$ & 74 & $64,9 \%$ & 40 & $35,1 \%$ & 197 & $67,9 \%$ & 93 & $32,1 \%$ \\
\hline
\end{tabular}

Preg.1 Las Infecciones de Transmisión Sexual

Preg.2 El SIDA es

Preg.3 EL VIH afecta a los linfocitos T CD4

Preg.4 La principal forma de transmisión de las Infecciones de Transmisión Sexual

Preg. 5 Las 3 principales formas de transmisión del VIH

Preg.6 Transmisión Sexual - Agente causal

Preg.7 Relación a los signos y síntomas de las ITS- VIH/SIDA

Preg.8 Infecciones de Transmisión Sexual - Agentes Patógenos

Preg.9 Infecciones de Transmisión Sexual - Lesiones

Preg.10 Fases del VIH como una Infección de Transmisión Sexual

Preg.11 Fase del VIH en la que aparecen los primeros síntomas y signos de la inmunodeficiencia

Preg.12 Las dos principales pruebas de laboratorio para diagnosticar que una persona es portadora del VIH

Preg.13 Diagnóstico de una persona portadora del VIH

Preg.14 Las principales pruebas de laboratorio para diagnosticar una Infecciones de Transmisión Sexual

Preg.15 Tratamiento de las Infecciones de Transmisión Sexual -VIH/SIDA

Preg.16 Complicaciones que trae las Infecciones de Transmisión Sexual-VIH/SIDA

Como se observa en la tabla 1 que contiene los ítems del instrumento y las respuestas de los estudiantes a cada uno de ellos, según facultad. Se ha consolidado en correcto e incorrecto. Con la finalidad de identificar, a través de los ítems, aquellos contenidos de mayor o menor conocimiento, se ha coloreado con verde aquellos que los estudiantes conocen más y con rojo los que conocen menos. En relación a los ítems que más conocen los estudiantes se observa que el $96.6 \%$ de ellos conocen el ítem 4 relacionado a la principal forma de transmisión de las ITS-VIH/SIDA, seguido del ítem 2 que aborda el concepto de SIDA con el $96.2 \%$; luego el $84.5 \%$ está el ítem 3 relacionado con el tipo de linfocito al que afecta el VIH, en este caso al T CD4. Siguiendo en forma decreciente con el $83.1 \%$ encontramos al ítem 1 relacionado a la definición de ITS. De los ítems restantes se aprecia que el ítem 5 relacionado a las 3 principales formas de transmisión de las ITS-VIH/SIDA alcanza el $80 \%$, seguido del $79.7 \%$ por el ítem 12 relacionado a prueba de laboratorio para identificar anticuerpos contra el VIH y por último con el $73.4 \%$ el ítem 14 relacionado a las principales pruebas de laboratorio para diagnosticar a las ITS. En relación a los ítems que menos conocen los estudiantes, se observa que son el 9, 15 y 7 referente a las lesiones, tratamiento, signos y síntomas de las ITS-VIH/SIDA, representado por el 100\%, 93.8\% Y 92.1\% de estudiantes. En cuanto la sintomatología que más desconocen es sobre gonorrea y chancroide, entre las más importantes, y la que más conocen es sobre el SIDA. A su vez, el tratamiento de las ITS-VIH/SIDA que más desconocen es sobre Tricomoniasis, Vaginosis bacteriana y la que conocen más es sobre la Sífilis. El ítem 11 referido a los signos y síntomas de 
inmunodeficiencia del VIH; luego el ítem 6 que está relacionado con el agente causal de las ITS con un $83.1 \%$. En orden decreciente continúan los ítems 10 y 16 con un $77.9 \%$ y 64.9\%, los cuales están referidos a las fases de la infección por VIH y las complicaciones de las ITS-VIH-SIDA. Tenemos a los ítems 13 y 8 que están relacionados con las pruebas de laboratorio para diagnosticar ser portador del $\mathrm{VIH}$ y a los virus y bacterias como agentes patógenos de las ITS con un $64.9 \%$ y $56.1 \%$ respectivamente. El agente patógeno de la ITS que más desconocen son del chancroide, tricomoniasis y el que más conocen es el agente patógeno del SIDA y herpes genital.

Tabla 2: Áreas críticas en el conocimiento sobre medidas preventivas de its - vih/sida en los estudiantes de la unslg, según facultad, 2017

\begin{tabular}{|c|c|c|c|c|c|c|c|c|c|c|c|c|c|c|c|c|}
\hline & \multicolumn{16}{|c|}{ Facultad } \\
\hline & \multicolumn{4}{|c|}{ Obstetricia } & \multicolumn{4}{|c|}{ Enfermería } & \multicolumn{4}{|c|}{ Medicina } & \multicolumn{4}{|c|}{ Total } \\
\hline & \multicolumn{2}{|c|}{ Incorrecto } & \multicolumn{2}{|c|}{ Correcto } & \multicolumn{2}{|c|}{ Incorrecto } & \multicolumn{2}{|c|}{ Correcto } & \multicolumn{2}{|c|}{ Incorrecto } & \multicolumn{2}{|c|}{ Correcto } & \multicolumn{2}{|c|}{ Incorrecto } & \multicolumn{2}{|c|}{ Correcto } \\
\hline & $\mathrm{N}$ & $\%$ & $\mathrm{~N}$ & $\%$ & $\mathrm{~N}$ & $\%$ & $\mathrm{~N}$ & $\%$ & $\mathrm{~N}$ & $\%$ & $\mathrm{~N}$ & $\%$ & $\mathrm{~N}$ & $\%$ & $\mathrm{~N}$ & $\%$ \\
\hline Preg.17 & 3 & $5,5 \%$ & 52 & $94,5 \%$ & 11 & $9,1 \%$ & 110 & $90,9 \%$ & 8 & $7,0 \%$ & 106 & $93,0 \%$ & 22 & $7,6 \%$ & 268 & 92, \\
\hline Preg.18 & 18 & $32,7 \%$ & 37 & $67,3 \%$ & 6 & $5,0 \%$ & 115 & $95,0 \%$ & 9 & $7,9 \%$ & 105 & $92,1 \%$ & 33 & $11,4 \%$ & 257 & 8 \\
\hline Preg.19 & 2 & $3,6 \%$ & 53 & $96,4 \%$ & 6 & $5,0 \%$ & 115 & $95,0 \%$ & 5 & $4,4 \%$ & 109 & $95,6 \%$ & 13 & $4,5 \%$ & 277 & Du, \\
\hline Preg.20 & 0 & $0,0 \%$ & 55 & $100,0 \%$ & 4 & $3,3 \%$ & 117 & $96,7 \%$ & 2 & $1,8 \%$ & 112 & $98,2 \%$ & 6 & $2,1 \%$ & 284 & 97 \\
\hline Preg.21 & 2 & $3,6 \%$ & 53 & $96,4 \%$ & 5 & $4,1 \%$ & 116 & $95,9 \%$ & 1 & $0,9 \%$ & 113 & $99,1 \%$ & 8 & $2,8 \%$ & 282 & 97,2 \\
\hline Preg.22 & 19 & $34,5 \%$ & 36 & $65,5 \%$ & 45 & $37,2 \%$ & 76 & $62,8 \%$ & 35 & $30,7 \%$ & 79 & $69,3 \%$ & 99 & $34,1 \%$ & 191 & $65,9 \%$ \\
\hline Preg.23 & 1 & $1,8 \%$ & 54 & $98,2 \%$ & 18 & $14,9 \%$ & 103 & $85,1 \%$ & 16 & $14,0 \%$ & 98 & $86,0 \%$ & 35 & $12,1 \%$ & 255 & 8 \\
\hline
\end{tabular}

Preg.17 Medidas Preventivas para evitar contraer una Infecciones de Transmisión Sexual-VIH/SIDA

Preg.18 La Abstinencia Sexual

Preg.19 El Sexo Seguro es una actividad sexual que no expone a la pareja a contraer alguna Infección de Transmisión Sexual

Preg.20 El preservativo constituye una medida preventiva para evitar contraer alguna Infecciones de Transmisión Sexual

Preg.21 Número de veces de uso, al terminar el acto sexual

Preg.22 Forma correcta de usar el preservativo

Preg.23 Las condiciones necesarias para usar un preservativo

En la Tabla 2 al igual que en el anterior se ha coloreado con verde aquellos ítems que los estudiantes conocen más y con rojo los que desconocen. Del total de estudiantes encuestados, es el ítem 20 relacionado al preservativo como medida preventiva de ITS, el que obtiene el mayor porcentaje de estudiantes que lo conoce, representado por el 97.9\%, con el 97.2\% está el ítem 21 relacionado sobre frecuencia del preservativo, le sigue el ítem 19 con un $95.5 \%$ relacionado a actividades que incluye el sexo seguro, luego el ítem 17 relacionado a medidas preventivas para evitar contraer ITSVIH/SIDA con el 92.4\%, el 18 sobre definición de abstinencia sexual con $88.6 \%$ luego el ítem 23 sobre las condiciones necesarias para usar el preservativo con $87.9 \%$. Por último el ítem 22 sobre procedimiento para el uso correcto del preservativo con el $63.9 \%$.

\section{DISCUSIÓN}

Del total los estudiantes encuestados se observa que en el $88.3 \%$ sus edades fluctúan entre 20 a 25 años que representan la etapa de adulto joven, seguido de $14.8 \%$ cuyas edades están en el rango de 16 a 19 años quienes son parte de lo denominado adolescencia de segunda fase. Con respecto al Sexo, se observa que el $79 \%$ estudiantes son de sexo femenino y el $21 \%$ masculino, es clara la predominancia del sexo femenino, que en Obstetricia representa $100 \%$ y $91.7 \%$ en Enfermería. En cuanto al Estado Civil se obtuvo que el 100\% (176) estudiantes son solteros. Estos datos concuerdan con el estudio realizado por Camacho (2013)(4) en estudiantes de la facultad de Medicina de la Universidad Nacional Mayor de San Marcos, que en relación al sexo del total de estudiantes encuestados el $61.8 \%$ son de sexo femenino. Siendo los estudiantes de Obstetricia y Enfermería con predominio de población femenina. Cabe mencionar que del total de los estudiantes encuestados el 100\% en el aspecto del estado civil eran solteros(as).
En cuanto a la relación que existe entre los conocimientos y prácticas de nuestros entrevistados, con un Chi cuadrado de Pearson $=0,00 ; p \leq 0.05$, se determina que si existe asociación entre ambas variables, apreciándose que los que tienen un conocimiento intermedio sobre epilepsia es $85,6 \%$, todos tienen prácticas adecuadas, con ello notamos una relación entre ambas variables.

En cuanto a la relación que existe entre los actitudes y prácticas de nuestros entrevistados, con un Chi cuadrado de Pearson= 0,$55 ; \mathrm{p} \geq$ 0.05 , se determina que no existe asociación entre ambas variables. rácticas, mas no entre conocimientos y actitudes ni actitud y práctica.

En referente al nivel de conocimientos sobre las ITS-VIH/SIDA y sus medidas preventivas en los estudiantes, en forma global, se observa que predomina el conocimiento medio en la facultad de Obstetricia presenta un mayor porcentaje con respecto a la facultad de Enfermería siendo esta de $81.8 \%$ y $76.8 \%$ respectivamente, mientras que en la facultad de Medicina predomina el nivel de conocimiento alto con un $45.6 \%$. El nivel de conocimiento alto está presente en porcentajes ínfimos como un 9.1\% en los estudiantes de Obstetricia y $4.2 \%$ en los estudiantes de Enfermería que a su vez presentan un elevado porcentaje de nivel de conocimiento bajo con un $19 \%$. Estos datos denotan un nivel de conocimiento superior de los estudiantes de Medicina con respecto a las otras facultades, a su vez los estudiantes de Obstetricia presentan un mejor nivel de conocimiento con respecto a los de Enfermería.

En cuanto a la dimensión del nivel de conocimientos sobre las ITS $\mathrm{VIH} / \mathrm{SIDA}$, se observa que predomina el nivel de conocimiento alto en estudiantes de Medicina con un $36.8 \%$, quienes comparados con sus pares de Enfermería y Obstetricia presentan mejor nivel de conocimientos ya que los estudiantes de dichas facultades 
presentan menos de $10 \%$ de nivel de conocimiento alto, siendo el porcentaje más ínfimo las de Enfermería con 1.7\%. En lo que respecta a la facultad de Obstetricia presenta mejor nivel de conocimiento comparado con la facultad de Enfermería ya que presenta un nivel de conocimiento bajo y medio de $50.9 \%$ y de $38.2 \%$ mientras que la facultad de Enfermería presenta un mayor porcentaje de nivel de conocimiento bajo siendo este de $78.5 \%$ y un $19.8 \%$ de nivel de conocimiento medio. Al respecto se presenta diversos estudios que contrastan estos resultados como la de Zelada y col. (2014)(1) en su estudio realizado en estudiantes de Enfermería en Cuba en la que pudo determinar que el nivel de conocimientos de los estudiantes es inadecuado con un $84,7 \%$. Además en el estudio realizado por Camacho (2013)6) se evidencio que los estudiantes de Obstetricia presentan un mejor nivel de conocimiento con respecto a estudiantes de Medicina y Enfermería. Siguiendo la siguiente distribución: Escuela de Obstetricia un $53.9 \%$ tienen un conocimiento alto, seguido de un $41 \%$ con conocimiento medio En la Escuela de Medicina Humana predomina el conocimiento medio con un $44.6 \%$, seguido del conocimiento alto con $43.2 \%$. En la Escuela de Enfermería un 50\% tiene un conocimiento medio, un $34.4 \%$ tienen un conocimiento alto. Sin embargo se cuenta con estudios en la cual los estudiantes de enfermería presentan un predominio de nivel de conocimiento alto, así por ejemplo Quispe y Ramos (2015) (5) en su estudio en estudiantes de la Facultad de Enfermería de la Universidad Nacional de Huancavelica" obtuvieron como resultado que un $79.7 \%$ de los estudiantes del VIII ciclo, tienen conocimiento sobre VIH/SIDA de nivel alto. Así mismo; de $16.5 \%$ estudiantes con conocimiento de nivel medio. De igual modo; de $3.8 \%$ estudiantes con conocimiento de nivel bajo. De igual forma Carhuayo y Figueroa (2013)(6) en su estudio en estudiantes de la Facultad de Enfermería de la Universidad Nacional de Ica; determinó que el nivel de conocimiento sobre VIH-SIDA fue alto alcanzando 99\%. En la dimensión del conocimiento sobre las medidas preventivas de las Infecciones de Transmisión Sexual -VIH/SIDA se observa que predomina el nivel de conocimiento alto en las tres facultades con un mayor porcentaje en los estudiantes de la facultad de Medicina de $80,7 \%$. En relación al nivel de conocimiento medio se observa que la facultad de obstetricia presenta mejor nivel de conocimiento que la facultad de enfermería con porcentajes de $20 \%$ y $16.5 \%$ respectivamente.

En forma global los conceptos que más conocen los estudiantes son la definición de SIDA, la principal forma de transmisión de las Infecciones de Transmisión Sexual, 3 principales formas de transmisión del VIH, las principales pruebas de laboratorio para diagnosticar una Infecciones de Transmisión Sexual, definición de las Infecciones de Transmisión Sexual y que el VIH afecta a los linfocitos $T$ CD4. Los conceptos que más desconocen los estudiantes son lo relacionado a las ITS y su agente patógeno, a los signos/síntomas de las mismas y la presencia de ulceras y secreciones según ITS así como el tratamiento de las ITS-VIH/SIDA Los ítems que más conocen relacionado a medidas preventivas son: el preservativo como medida preventiva de ITS, el que obtiene el mayor porcentaje de estudiantes que lo conoce, seguido muy de cerca sobre frecuencia del preservativo, le sigue el ítem relacionado a actividades que incluye el sexo seguro, luego el ítem relacionado a medidas preventivas para evitar contraer ITSVIH/SIDA y las condiciones necesarias para usar el preservativo. Por último, el ítem 22 sobre procedimiento para usar el preservativo es el ítem que menos conocen con un $69.3 \%$ en los estudiantes de Medicina, un $65.5 \%$ y $62.8 \%$ de estudiantes que lo conocen de la facultad de Obstetricia y Enfermería respectivamente.

Estos datos son contrastados con diversos estudios similares realizados, así por ejemplo Céspedes et. al. (2015) (2)) realizo un estudio en estudiantes de Medicina en Cuba donde evidencio que poseen un bajo nivel de conocimientos sobre las ITS, baja percepción de riesgo y una conducta sexual no responsable que los hace susceptibles de contraer estas infecciones. Los estudiantes encuestados identificaron al VIH, sífilis y gonorrea en mayor medida que el resto de las ITS. Mirabal et. al. (2013) (3) en su estudio realizado en estudiantes de Medicina en Cuba demostró que el componente cognitivo sólo un 46 \% supo reconocer las vías de transmisión del VIH/SIDA, y un 13,2 \% no identificó las manifestaciones clínicas relacionadas con las infecciones de transmisión sexual, con respecto a la identificación de las manifestaciones clínicas relacionadas con las ITS el 67.5 \% mostró un conocimiento valorado de muy adecuado, un $19.3 \%$ de adecuado y un $13.2 \%$ de inadecuado. Camacho (2013) (6) demostró que en la Escuela de Medicina Humana entre los aspectos que no conocen son los signos y síntomas ITS en varones con $55.4 \%$, signos y síntomas ITS en mujeres y sobre el uso adecuado del condón con $54.1 \%$, agente causal con $52.7 \%$, y entre los aspectos que menos erraron tenemos sobre el agente causal del SIDA con 6.7\%, Información de las ITS con 8.1\%. En la Escuela de Obstetricia entre los aspectos que no conocen tenemos sobre el agente causal de ITS con $76.9 \%$, signos y síntomas ITS en varones con $74.3 \%$ y entre los aspectos que menos erraron tenemos acerca de la información de ITS con $0 \%$, agente causal del SIDA con $2.5 \%$, medidas preventivas para evitar una ITS con 7.6\%. En la Escuela de Enfermería entre los aspectos que no conocen tenemos acerca del uso adecuado del condón con $75 \%$, signos y síntomas ITS en varones con $68.7 \%$, agente causal de ITS con $62.5 \%$, diagnostico de ITS con $59.8 \%$ y entre los aspectos que menos erraron tenemos acerca del agente causal del SIDA con 3.1\%, Información de ITS y clasificación de ITS con 12.5\%. A su vez Quispe y Ramos (2015) (5) en su estudio en estudiantes de la Facultad de Enfermería de la Universidad Nacional de Huancavelica" obtuvieron como resultado que un $57.0 \%$ estudiantes del VIII ciclo tuvieron conocimiento medio sobre formas de transmisión de VIH/SIDA. Al mismo tiempo Carhuayo y Figueroa (2013) (6) obtuvo como resultado que la variable nivel de conocimiento sobre los aspectos generales del VIH-SIDA como concepto, fisiopatología y síntomas fue alto alcanzando un $98 \%$ y solo el $2 \%$ fue medio, sobre las vías de transmisión del VIH-SIDA fue medio alcanzando un $68 \%$, sobre los factores de riesgo del VIH-SIDA fue alto alcanzando un $81 \%$; sobre el diagnóstico y tratamiento del VIH-SIDA fue alto alcanzando un $51 \%$ y el nivel de conocimiento sobre prevención del VIHSIDA fue alto alcanzando un $76 \%$. 


\section{BIBLIOGRAFÍA}

1. Zelada A, Valdivia A, Saavedra C, Oliva M, González M. Nivel de conocimientos sobre las Infecciones de Trasmisión Sexual (ITS) y conducta en adolescentes estudiantes de Enfermería. Rev. Cubana de Medicina Tropical 2014; 66(2): 1-8.

2. Céspedes G., Pupo C., Ramírez C., Ballester R. Las infecciones de transmisión sexual y los estudiantes de Medicina. Rev. Médica Multimed 2015; 19(1) 1-16.

3. Mirabal N., Betancourt B., Prieto C., Fernández F. Actitudes socioculturales frente a las infecciones de transmisión sexual en estudiantes de Medicina. Humanidades Médicas en Cuba 2013; 13(1), 56-71.

4. Camacho J. Relación entre el nivel de conocimientos sobre las infecciones de trasmisión sexual (ITS) y la actitud hacia conductas sexuales de riesgo en estudiantes de la Facultad de Medicina UNMSM, 2013. . [Tesis para optar el título de licenciada en Enfermería]. Universidad Nacional Mayor de San Marcos Lima Perú, 2013.

5. Quispe Y., Ramos K. Conocimiento sobre VIH-SIDA y actitud frente al uso de preservativo en estudiantes de la Facultad de Enfermería de la Universidad Nacional de Huancavelica, 2015. [Tesis para optar el título de licenciada en Enfermería]. Universidad Nacional de Huancavelica-Perú, 2015.

6. Denisse L., Campos S. Nivel de conocimientos sobre las infecciones de transmisión sexual-VIH/SIDA en los estudiantes de enfermería de la Universidad Nacional San Luis Gonzaga de Ica, 2013. [Tesis para optar el título de licenciada en Enfermería]. Universidad Nacional San Luis Gonzaga de Ica, Perú 2013.

7. Fernández F., Rodríguez C., González S., Aranda G, Riego A. et. al. Nivel de conocimientos sobre sexualidad en estudiantes de carreras de ciencias de la salud de la universidad veracruzana. Universalud 2013; 17(9): 12-18.

8. Rojas S. Nivel de Conocimientos sobre Infecciones de Transmisión Sexual en madres adolescentes del Centro de Salud Chilca - Huancayo 2013. [Tesis para optar el título de licenciada en Obstetricia]. Universidad Peruana del Centro Huancayo-Perú; 2016. 9. Lucano P., Clarita M. Conocimientos y prácticas sobre sexualidad en estudiantes universitarios del primer y quinto semestre de la escuela académico profesional de Obstetricia. Abril 2014. [Tesis para optar el título de licenciada en obstetricia]. Universidad Nacional Mayor de San Marcos, Lima-Perú; 2014.
10. Sulca S. y Bethsabe S. Relación entre el nivel de conocimientos sobre las infecciones de trasmisión sexual (ITS) y la actitud hacia conductas sexuales de riesgo en gestantes adolescentes del hospital nacional Daniel Alcides Carrión - mayo del 2014. [Tesis para optar el título de licenciada en obstetricia]. Universidad Nacional Mayor de San Marcos, Lima-Perú; 2014.

11. Paz C., Rodríguez A. Conocimientos y comportamientos en salud sexual de estudiantes indígenas guatemaltecos residentes en una comunidad rural. Revista Cubana de Medicina General Integral 2014; 30(4):467-480.

12. Córdova K. Nivel de Conocimiento sobre VIH/SIDA y manifestaciones de la sexualidad en alumnos del quinto año de secundaria de la I. E. José Toribio Polo, Ica-Perú noviembre 2013. [Tesis para optar el título de licenciada en Enfermería]. Universidad Privada San Juan Bautista de Ica, Perú 2013.

13. Carhuayo R., Figueroa M. Relación entre el nivel de conocimiento y actitud sobre VIH-SIDA de los estudiantes de la facultad de Enfermería - UNICA 2013. Rev. Enfermería a la Vanguardia, 2014: 2 (1); 15-25.

14. Folch C., Alvarez L., Casabona J., Brotons M., Castellsagué X. Determinantes de las conductas sexuales de riesgo en jóvenes de Cataluña. Rev. Esp. Salud Pública 2015; 89: 471-485.

15. Forcada P., Pacheco S., Pahua E., Pérez P., Todd E. et. al. Conducta sexual de riesgo en estudiantes universitarios: factores de riesgo y protección. Revista Intercontinental de Psicología y Educación México 2013; 15(1): 23-46.

16. Bagnato M., Jenaro C., Flores N., Guzmán K. Factores culturales asociados a las conductas sexuales en estudiantes universitarios de Uruguay y España: Estudio Preliminar. Psicología, conocimiento y sociedad 2014; 1: 6 - 32

17. Morales S., Arboleda O., Segura A. Las prácticas sexuales de riesgo al VIH en población universitaria. Rev. Salud Pública Medellin Colombia 2014; 16 (1): 27-39.

18. Alejandro O., López J. Ejercicio de la sexualidad en estudiantes de pregrado. Rev. Psicología y Salud, México 2015; 25(2): 233-241.

19. Granados R., Sierra C. Excitación sexual: una revisión sobre su relación con las conductas sexuales de riesgo. Terapia Psicológica 2016; 34(1), 59-70.

20. Saeteros R., Pérez J., Sanabria G., Díaz Z. Efectividad de una estrategia de educación sexual para universitarios ecuatorianos. Rev. Cubana salud pública. 2016; 42(4): 1-15. 